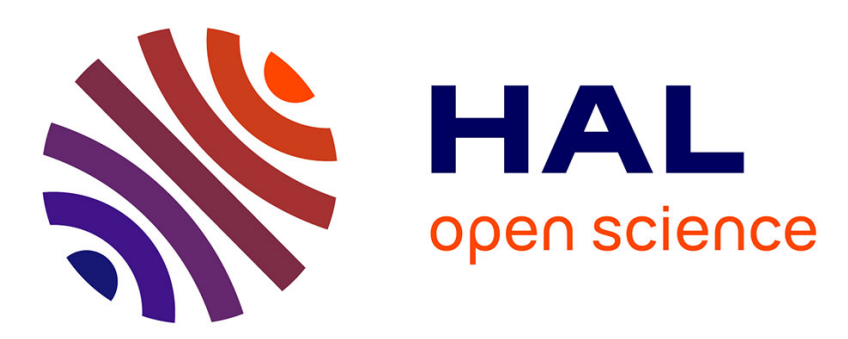

\title{
On the effects of an imposed magnetic field on the elliptical instability in rotating spheroids
}

\author{
Wietze Herreman, Michael Le Bars, Patrice Le Gal
}

\section{To cite this version:}

Wietze Herreman, Michael Le Bars, Patrice Le Gal. On the effects of an imposed magnetic field on the elliptical instability in rotating spheroids. Physics of Fluids, 2009, 21, pp.046602. 10.1063/1.3119102 . hal-00426018

\section{HAL Id: hal-00426018 https://hal.science/hal-00426018}

Submitted on 23 Oct 2009

HAL is a multi-disciplinary open access archive for the deposit and dissemination of scientific research documents, whether they are published or not. The documents may come from teaching and research institutions in France or abroad, or from public or private research centers.
L'archive ouverte pluridisciplinaire HAL, est destinée au dépôt et à la diffusion de documents scientifiques de niveau recherche, publiés ou non, émanant des établissements d'enseignement et de recherche français ou étrangers, des laboratoires publics ou privés. 


\title{
On the effects of an imposed magnetic field on the elliptical instability in rotating spheroids
}

\author{
W. Herreman, M. Le Bars \& P. Le Gal \\ March 17, 2009 \\ Institut de Recherche sur les Phénomènes Hors Équilibre \\ CNRS UMR 6594 and Aix-Marseille Université \\ 49 rue F. Joliot Curie, B.P. 146, F-13384 Marseille Cedex 13, France
}

\begin{abstract}
The effects of an imposed magnetic field on the development of the elliptical instability in a rotating spheroid filled with a conducting fluid are considered. Theoretical and experimental studies of the spin-over mode, as well as a more general short-wavelength Lagrangian approach, demonstrate that the linear growth rate of the instability and the square amplitude of the induced magnetic field fall down linearly with the square of the imposed magnetic field. Application of the results to the Galilean moon Io confirms the fundamental role played by the elliptical instability at the planetary scale.
\end{abstract}

\section{Introduction}

The elliptical instability is a generic instability of rotating flows with elliptical streamlines. It has for instance been observed in wakes [1], [2], in elliptically deformed containers, [3], [4], [5], [6], and more generally in the transition to turbulence of strained vortices [7]. Since its discovery in the mid-1970s, it has received considerable attention, theoretically, experimentally and numerically (see for instance the review by Kerswell [8]).

Flows with elliptical streamlines arise as a superposition of rotation and a small strain field, and the instability mechanism has been identified as a 
parametric resonance of pairwise inertial waves coupled by this strain [9], [10]. In the geophysical context of liquid planetary cores [11],[12], [13], the strain comes from the tidal deformations due to gravitational interaction between neighboring celestial bodies. The elliptical instability (also called tidal instability in this context), as well as the closely related precessional instability, may leave traces in the gravitational and magnetic fields of planets [14], [13], and may even provide alternative sources to power the geodynamo [11], [15]. Even if the hydrodynamic of the elliptical instability is today well known, its planetary consequences are still controversial and necessitate a full understanding of the magnetohydrodynamic (MHD) of the elliptical instability, which remains a mostly open question (e.g. [16]). Understanding the MHD of the elliptical instability is also important in metallurgic applications, especially regarding its role in the transition from two to three-dimensional MHD-turbulence [17].

In the present paper, we consider an elliptically deformed rotating sphere filled with a conducting fluid (figure 1) and we study both theoretically and experimentally the effects of an imposed magnetic field parallel to the rotation axis on the development of the elliptical instability. This situation is reminiscent of planetary configurations where a tidally deformed moon with a liquid iron core rotates in the magnetic field of its planet, as for instance the Galilean moon Io in the vicinity of Jupiter. Our purpose is to answer the two following questions. How is the elliptical instability damped by the magnetic field? And what is the amplitude of the magnetic field induced by the elliptical instability?

This article, which completes and extends the previous works of Lacaze et al. [16] and Thess \& Zikanov [17], is organized as follow. We first focus on the so-called spin-over mode, which corresponds to the simplest mode of the elliptical instability in spheroids, excited at the smallest values of the Reynolds number above threshold. We derive a nonlinear and viscous model of its development under an imposed magnetic field valid for low values of the magnetic Reynolds number, based on the hydrodynamical model of Lacaze et al. [5] and including the magnetic damping term determined by Thess \& Zikanov [17]. These results are validated experimentally using an extended version of the set-up of Lacaze et al. [16], with stronger imposed magnetic fields. These results are then extended to the large magnetic Reynolds number, large Reynolds number limit relevant to planetary applications, using a short-wavelength Lagrangian theory [18]. An analytical expression of the growth rate of the elliptical instability is determined and results are finally 
applied to the case of Io, highlighting the importance of the elliptical instability at the planetary scale.

\section{Set-up and state of the art}

We consider the experimental system sketched in figure 1. A spherical cavity with radius $R$, molded in a deformable silicone block, is filled with a liquid metal, with permeability $\mu$, conductivity $\sigma_{e}$, kinematic viscosity $\nu$, and density $\rho$. It is set in rotation at a constant angular velocity $\Omega_{0}=\Omega_{0} \hat{\mathbf{z}}$ around the vertical z-axis, and a homogenous magnetic field $\mathbf{B}_{0}=B_{0} \hat{\mathbf{z}}$ is externally imposed along the same axis with a pair of Helmholtz coils. Following the original idea of Malkus [3], a pair of fixed and opposed rollers compresses the transverse section of the deformable container, giving it an elliptical crosssection with long axis $R \sqrt{1+\epsilon}$ along $x$ and short axis $R \sqrt{1-\epsilon}$ along $\mathrm{y}$, $\epsilon$ being the eccentricity of the elliptical deformation. Previous experimental studies (e.g. [5]) have demonstrated that the flow in the volume effectively exhibits elliptical streamlines due to the boundary deformation and approaches the theoretical elliptical base-flow

$$
\mathbf{U}_{\mathbf{b}}=\left(-\Omega_{0} \sqrt{\frac{1+\epsilon}{1-\epsilon}} y, \Omega_{0} \sqrt{\frac{1-\epsilon}{1+\epsilon}} x, 0\right) .
$$

Together with a homogenous magnetic field along the axis, this flow defines an exact base state of the MHD equations on which perturbations may grow due to the elliptical instability. In our experiments, the magnetic Reynolds number $R m=\Omega_{0} R^{2} / \eta$, where $\eta$ is the magnetic field diffusion $\eta=\left(\sigma_{e} \mu\right)^{-1}$, is small $\left(R m=O\left(10^{-2}\right)\right)$ and magnetic field diffusion is always dominant over magnetic field advection. In this limit, the scales

$$
\begin{gathered}
{[\mathbf{r}]=R \quad[t]=\Omega_{0}^{-1} \quad[\mathbf{u}]=\Omega_{0} R} \\
{[p]=\rho\left(\Omega_{0} R\right)^{2} \quad[\mathbf{b}]=R m B_{0}}
\end{gathered}
$$

respectively for space, time, velocity, pressure and magnetic field, are well adapted to non-dimensionalize the perturbation problem. In addition to $R m$, the relevant non-dimensional parameters are the Ekmann number $E=$ $\nu / \Omega_{0} R^{2}$, which measures the importance of diffusive effects over inertial terms, and the Elsasser number $\Lambda=\sigma_{e} B_{0}^{2} / \rho \Omega$, measuring the ratio of Lorentz 
force effects over the inertial forces. In our experiments, $E=O\left(10^{-4}\right)$ and $\Lambda \leq O(1)$ typically.

The hydrodynamic stability of elliptical flow in spheroids was previously studied in [5], which formalizes an asymptotic theory in terms of inertial wave coupling. In the limit $\Lambda \sim \epsilon, R m \rightarrow 0$, this theory can be extended to include the magnetic field effect perturbatively. We will not go as far, as the combination of the results of [5], [16] and [17], allows us to describe the linear and nonlinear dynamics of the dominant spin-over mode, which is the only mode accessible to purely hydrodynamical experiments using the present device in a spherical geometry with a fixed strain field [19]. The spinover mode is mainly a solid body rotation around an inclined axis, whose horizontal projection $\boldsymbol{\Omega}_{\mathbf{H}}$ is aligned with the axis stretched by the strain field, at polar angle in the vicinity of $-45^{\circ}$ in the $(x, y)$ plane (see figure 1). A low-dimensional model was derived in [5] in close agreement with the experiments, which describes the nonlinearly purely fluid evolving spin-over mode as a solid body rotation. Even though $E$ is small in the experiment, viscosity plays an essential role. Indeed, it postpones the elliptical instability to a critical eccentricity and allows the nonlinear dynamical system to have stable non-trivial fixed points.

Stays the question whether the spin-over mode remains the most unstable mode in presence of a magnetic field, which seems hard to answer without a more complex global analysis of the elliptical instability. In [17] (see also section 5), the local growth rates of elliptical instability in an unbounded domain were calculated using Flocquet theory. For the limit of small $\epsilon$ we are interested in, asymptotic arguments as in [10] imply a growth rate linear in $\epsilon$ and a magnetic damping $\gamma_{M}=\Lambda \lambda^{2} / 4$, where $\lambda$ is the wave frequency in the rotating frame. Since all elliptically interesting waves have $\lambda \simeq 1$ [5], [19], local theory indicates that the magnetic field damping acts similarly on all couplings, no matter what their spatial structure is. Since in our device the spin-over mode is always the most unstable mode in the hydrodynamical experiments, we expect that it remains the case when a magnetic field is imposed.

Thess and Zikanov [17] also extended the non-linear, inviscid model of the spin-over dynamics to include the magnetic field effects in the low $R m$ limit. They found that the magnetic field introduces a Joule damping, which only operates on the rotations with axis transverse to the imposed magnetic field, identically to the Joule damping of solid conductors rotating in a strong magnetic field, commonly used in magnetic brakes. In our experimental set- 
up, both viscous and magnetic field effects are important. The eccentricity is small $\epsilon \simeq 0.1$, which means that $\Lambda$ is at most of order $O\left(10^{-1}\right)$ in the experiments where we observe the instability. The important consequence is that the magnetic field in this case is always too small to change the viscous boundary layer into a Hartmann-layer, so that there is no need of a more complex boundary layer analysis. This also implies that the viscous terms in the nonlinear system of [5] may be used here. Finally, there will be no significant contributions to the external and internal magnetic fields due to the boundary layer, which would make the field deviate from the field induced by the non-viscous spin-over mode, calculated in [16].

Notice that all these suppositions will be confirmed a-posteriori by the good agreement between the following theory and our experimental results.

\section{Analytical study of the spin-over mode}

Combining the results of [5] and [17], the nonlinear evolution of the spinover mode can be modeled in the laboratory frame of reference as a solid body rotation with angular velocity $\Omega=\left(\Omega_{1}(t), \Omega_{2}(t), \Omega_{3}(t)\right)$, which evolves according to the nonlinear system

$$
\begin{aligned}
& \dot{\Omega}_{1}=-\frac{\epsilon}{2-\epsilon}\left(1+\Omega_{3}\right) \Omega_{2}-\left(\gamma_{s o}+\Lambda / 4\right) \Omega_{1}, \\
& \dot{\Omega}_{2}=-\frac{\epsilon}{2+\epsilon}\left(1+\Omega_{3}\right) \Omega_{1}-\left(\gamma_{s o}+\Lambda / 4\right) \Omega_{2}, \\
& \dot{\Omega}_{3}=\epsilon \Omega_{1} \Omega_{2}-\gamma_{3} \Omega_{3}+\nu_{n l}\left(\Omega_{1}^{2}+\Omega_{2}^{2}\right) .
\end{aligned}
$$

On the right hand sides, we first recognize the destabilizing terms from the non-viscous system. The damping of the spin-over mode is controlled by the viscous linear boundary layer $\gamma_{s o}=2.62 \sqrt{E}$ in the horizontal directions and $\gamma_{3}=2.85 \sqrt{E}$ around the vertical axis. Supplementary nonlinear terms arise through the boundary layer, $\nu_{n l}=1.42 \sqrt{E}$. All these coefficients are explicitly detailed in Lacaze et al. [5] and find their origin in the classical analysis of Greenspan [20]. The magnetic field only adds a linear term corresponding to the Joule-damping $\Lambda / 4$ in the directions perpendicular to the imposed field. The terms due to the viscous frequency detuning are left out from the model as in [5], since they only introduce negligible differences in the limit of small Ekman numbers we are interested in. 
Linearising the system around the trivial fixed point $\mathbf{0}$, we calculate the linear growth rate of the spin-over mode

$$
\sigma=\frac{\epsilon}{\sqrt{4-\epsilon^{2}}}-2.62 \sqrt{E}-\Lambda / 4
$$

In agreement with [17], the magnetic damping lowers the growth rate of the spin-over mode linearly with $\Lambda$, and the system becomes stable above a critical Elsasser number

$$
\Lambda^{c}=4\left(\frac{\epsilon}{\sqrt{4-\epsilon^{2}}}-2.62 \sqrt{E}\right) .
$$

Some time-series for the horizontal projection of the spin-over mode amplitude, $\Omega_{H}=\sqrt{\Omega_{1}^{2}+\Omega_{2}^{2}}$, found by numerical integration of the nonlinear system (3)-(5), are shown in figure 2(a). After an exponential growth, the flow always goes towards a stable non-zero fixed point which is a stable focus. Before saturation the spin-over mode horizontal amplitude displays a small overshoot which originates from the spiral trajectory around this focus. For increasing magnetic field amplitudes, both the linear growth rate and the saturation amplitude decrease. The non-zero fixed points of (3) can be calculated explicitly. The square of the spin-over mode amplitude at saturation writes

$$
\Omega_{H}^{2}=4 \frac{\gamma_{3}}{\epsilon} \frac{\sigma}{\epsilon-4 \nu_{n l} / \sqrt{4-\epsilon^{2}}} .
$$

Note that $\Lambda$ only appears in this formula through the growth rate. According to [16], the field induced by the non-viscous spin-over mode at low $R m$ is a dipole with axis transverse to the imposed field, in quadrature with the rotation axis of the spin-over mode. On the dipole axis outside the spheroid, the field is purely radial and decays as

$$
b_{r}=\frac{\left|\Omega_{H}\right|}{35} \frac{1}{r^{3}} .
$$

Combining (8) and (9), we expect a linear decrease of the square of the induced field amplitude with $\Lambda$ at fixed $\epsilon$ and $E$. The polar angle in the $(x, y)$ plane of the saturated spin-over axis is determined by

$$
\bar{\phi}_{s o}= \pm \arctan \left(\frac{\Omega_{2}}{\Omega_{1}}\right)= \pm \arctan \left(-\sqrt{\frac{2-\epsilon}{2+\epsilon}}\right)
$$


so that the vorticity of the saturated spin-over mode is not exactly aligned with the direction of maximum stretching at $-45^{\circ}$ of the long axis of the spheroid (e.g. $\bar{\phi}_{\text {so }}=-42.1^{\circ}$ for $\epsilon=0.10$ ).

Lacaze et al. [5] tested experimentally the purely hydrodynamical version of this theory and found good agreement for both linear and nonlinear stages. However, the nonlinear overshoot was not observed and the authors noticed that the experimental saturation amplitudes of the spin-over mode agree better with the maximum theoretical amplitudes attained during the overshoot than with the theoretical saturation amplitudes. We now extend these experimental results by taking the magnetic field into account.

\section{Experiments}

Our experimental set-up is an extension of the one presented in [16] (see figures 1 and 3). The experimental parameters are $R=22.75 \mathrm{~mm} \Omega_{0} \in$ $[0,10 \pi] \mathrm{rads}^{-1}$ and $\epsilon=0.10$. The imposed field $B_{0}$ ranges between 0 and $0.13 T$ (up to 100 times larger than in the previous set-up [16]). It is realized with a set of watercooled copper Helmholtz coils, powered by a stabilized DCsupply. The liquid metal we use is Galinstan, a gallium-indium-tin eutectic liquid at room temperature, with $\rho=6440 \mathrm{~kg} \mathrm{~m}^{-3}, \nu=9.5 \times 10^{-5} \mathrm{~m}^{2} \mathrm{~s}^{-1}$, $\sigma_{e}=2.9 \times 10^{6} \mathrm{~S} \mathrm{~m}^{-1}$ and $\mu=\mu_{0}=4 \pi \times 10^{-7} \mathrm{~T}^{-1} \mathrm{~m}$. According to [16], the field induced by the non-viscous spin-over mode at low $R m$ is a dipole with axis transverse to the imposed field, in quadrature with the rotation axis of the spin-over mode. It is measured in the experiment by a radial Hall-probe mounted in the equator plane of the spheroid, facing the compressed direction at a polar angle of $45^{\circ}$. The probe is $26.5 \pm 0.5 \mathrm{~mm}$ away from the center of the sphere. The hall probe and the Gaussmeter have a maximum sensibility of $s=300 \mu \mathrm{T} / \mathrm{mV}$. Since the induced fields are of order $O\left(10^{-4} B_{0}\right)$, the probes are used at the limit of their sensibility. This implies careful positioning, thorough prefiltering and amplification of the recorded signals. In practice, the electric signal produced by the Hall-probe is put to zero before each experimental run. The recorded signals are prefiltered with a low-pass filter at $f_{c}=2 \mathrm{~Hz}$, and amplified by a factor 50 . The signals are transferred to the data-acquisition unit on the laboratory computer.

Figure 2(b) shows the experimentally recorded radial components of the induced magnetic fields for different $\Lambda$. The shapes and relative positions of the experimental records compare well with the theoretical profiles of figure 
2(a), with an exponential growth (see also figure 4) and a slight overshoot preceding a saturation at a constant level. As expected, there is a gradual decrease of growth rates and saturation amplitudes with $\Lambda$. Notice again that the amplitude overshoot was not observed in the purely hydrodynamical experiments (see [5]). At $\Lambda=0.121$ and higher, we continue to observe a nonzero induced magnetic field, but it becomes increasingly difficult to determine a true exponential growth. These fields probably come in our experimental set-up from misalignment between the axis of rotation of the sphere, the axis of the rollers inducing the elliptical deformation, and the axis of the imposed field.

The signals also give us quantitative informations on the growth rates and saturation amplitudes. As shown in figure 5, the growth rate decreases as $\Lambda / 4$, following the analytical result given by (6). Also shown are the theoretical dashed curves for $\epsilon=0.095$ and $\epsilon=0.105$ representing the uncertainty in $\epsilon$. As can be observed, the experimental data are in complete agreement with the theory within this $5 \%$ error range, without any adjustment parameter. Figure 6 shows that the square of the induced field $b_{r}^{2}$ behaves as the growth rate, in close agreement with the theory (see formula 8 and 9). Figure 6 also provides an experimental measurement of the critical Elsasser number $\Lambda^{c} \simeq 0.096$, close to the theoretical value $\Lambda^{c}=0.103$.

Using (9), we systematically translate the magnetic field measurements to spin-over-mode amplitude and show in figure 7 the variations of the ratio $\zeta_{1}$ of experimental saturation amplitudes to theoretical saturation amplitudes. The ratio $\zeta_{1}$ significantly decreases with the Elsasser number, where we expected a constant value close to 1 . This discrepancy between theoretical and experimental saturation values was already observed in the absence of magnetic field in [5]. Several explanations can be provided. From a theoretical point of view, all nonlinear viscous corrections as well as possible secondary instabilities are not included in our model but may become important, especially far from the linear instability threshold. And from an experimental point of view, one can notice that measurements at a fixed $-45^{\circ}$ angle only take into account a fraction of the spin-over amplitude when $\bar{\phi}_{s o} \neq-45^{\circ}$, this effect being also more important far from the linear instability threshold.

Lacaze et al. [5] also remarked that the ratio $\zeta_{2}$ of experimental saturation amplitudes to the maximal theoretical amplitudes attained during the overshoot remains constant over a rather large range of Ekman number. As shown in figure 7 , this remain valid over a large $\Lambda$-interval. We expect that this behavior is not a coincidence, but that it could be revealed by a more 
sophisticated model, beyond the scope of this paper. However, and contrary to the hydrodynamical experiments by [5] where $\zeta_{2} \sim 1$, theoretical predictions always underestimate the experimental measurements by a factor 1.42 in our case. Possible explanations are error in the positioning of the probe (the field decreases rapidly in $r^{-3}$ ), but most probably uncertainties in the value of the electrical conductivity $\sigma_{e}$ of Galinstan (values in the literature typically range between 2.3 and $3.5 \times 10^{6} \mathrm{~S} \mathrm{~m}^{-1}$ ). Note also that the elliptical deformation of the spheroid as well as the misalignment between the spin-over axis and the axis of maximum strain are not taken into account in equation (9).

\section{From laboratory models to geophysical ap- plications}

Magnetic induction by inertial waves is of particular interest in geo- and astrophysical applications. For instance, Kerswell and Malkus [13] have suggested that Io's magnetic field is induced from Jupiter's magnetic field by tidally driven inertial waves resonance, without dynamo action. However, our previous results derived in the limit of dominant magnetic diffusion (i.e. low $R m$ ) and for the laminar spin-over mode (i.e. at rather large $E$ ) cannot apply directly to planetary configurations, corresponding to the limit of small $E$, large $R m$ and probably large wavenumbers. As can be seen in the visualizations of figure 8 , the flow can then become increasingly complex, especially at small scale, and an extension of our analysis is necessary. Fortunately, a more general expression of the growth rate of the tidal instability, independent of the geometry of the flow, can be derived using the so-called local approach. Our goal here is not to give the exact expression of the growth rate of the various modes explicitly excited in a given planet but to determine an analytical expression able to describe the power dependence of the growth rate on all dimensionless numbers and to determine an order of magnitude of the various prefactors.

The local approach is based on the inviscid short-wavelength Lagrangian theory developed in [9] and [21], then generalized in [22] and [18]. There, perturbations are assumed to be sufficiently localized in order to be advected along flow trajectories and are searched as local plane waves of the form

$$
(\mathbf{u}, p)=(\mathbf{u}(t), p(t)) e^{i \mathbf{k}(t) \cdot \mathbf{x}},
$$


where $\mathbf{k}(t)$ is the time-dependent wave vector and $\mathbf{x}$ the position vector. This method has been applied to the elliptical instability by Le Dizès [23]. Here, we extend his results by taking into account the induction equation and the Lorentz force in the limit of small Elsasser number (e.g. $\Lambda \leq O(\epsilon)$ ) in the presence of an imposed vertical magnetic field $B_{0}$, looking for a perturbed magnetic field under the same wave form

$$
\mathbf{b}=\mathbf{b}(t) e^{i \mathbf{k}(t) \cdot \mathbf{x}}
$$

Details of the analysis are given in the appendix. Notice that in the following, we do not consider the limit of small magnetic Reynolds number anymore; hence, the magnetic field is made dimensionless using the amplitude of the imposed field $B_{0}$ rather than $R m B_{0}$ as in the previous sections. MHD equations are solved analytically using a perturbative expansion in eccentricity $\epsilon$, supposing that the Lorentz force is of order $\epsilon$. In this context, equations for fluid motions at order 0 are similar to the purely hydrodynamical case. Through the Lorentz force, the magnetic field induces a correction in the fluid equation at order 1 , hence a correction in the growth rate of the instability.

Using the $2 \mathrm{D}$ base flow $\mathbf{U}_{\mathbf{b}}$ given by (1), which corresponds to a stationary tidal deformation, we find a non viscous growth rate

$$
\sigma_{n v}=\frac{9}{16} \epsilon-\frac{k^{4} \Lambda}{4\left(R m^{2}+k^{4}\right)}
$$

where $k$ is a constant equal to the norm of the wave vector $\mathbf{k}(t)$ at leading order in $\epsilon$ (see equation 29 in appendix). The viscous damping rate resulting from the boundary layer can be estimated following [5], and induces a supplementary correction of the order $O\left(E^{1 / 2}\right)$. Notice that in the limit of small magnetic Reynolds number, one immediately finds the linear Joule damping $-\Lambda / 4$ determined in section 3 . The present result generalizes the validity of this scaling to all possible excited modes of the elliptical instability. Note also that the numerical factor before $\epsilon$ is different from (6), but remains of the same order of magnitude in the relevant limit of small $\epsilon$.

The previous result can still not be directly applied to the case of Io, where the elliptical deformation is not stationary. Indeed, as explained for instance in [13], Io is almost synchronized in its revolution around Jupiter, but orbital resonances with Europa and Ganymede force it to follow a slightly elliptical orbit of eccentricity 0.004 . As a result, the tidal bulge raised by Jupiter, of magnitude $\epsilon \sim 6 \times 10^{-3}$, does not rotate exactly at the same velocity as Io's 
spin, but oscillates back and forth across Io's body with a typical angular velocity $1-\beta \cos (t)$, where $\beta \sim 0.008$ is twice the eccentricity of Io's orbit and where time is made dimensionless using Io's spin velocity. In this case, the base flow in Io's core at first order in $\beta \epsilon$ writes (see Appendix)

$$
\begin{aligned}
\mathbf{U}_{\mathbf{b}}= & (-y+\beta \epsilon \cos (t)(\sin (2 t) x-\cos (2 t) y), \\
& x-\beta \epsilon \cos (t)(\cos (2 t) x+\sin (2 t) y]), 0),
\end{aligned}
$$

and the growth rate of the elliptic instability writes

$$
\sigma=\frac{17}{64} \sqrt{(\beta \epsilon)^{2}-\frac{576}{289} \frac{\Lambda^{2} R m^{2} k^{4}}{\left(R m^{2}+4 k^{4}\right)^{2}}}-\frac{3}{4} \frac{k^{4} \Lambda}{R m^{2}+4 k^{4}} .
$$

Formula (15) is closely related to (13), where the eccentricity $\epsilon$ in the case of a stationary tidal deformation has been replaced by the product of the tidal bulge times the amplitude of the perturbation $\beta \epsilon$. In particular at small $R m$, we once again end up at first order with a Joule damping linear in $\Lambda$. As mentioned before, surface viscous effects induce a correction to this formula of order $O\left(E^{1 / 2}\right)$ that could be explicitly determined. This is not done here, since the interest of formula (15) is to determine the relevant power law dependence on all dimensionless parameters (i.e. $\epsilon, R m, \Lambda, E$ ) as well as the order of magnitude of the various prefactors. In the following, we use for illustration the explicit values shown in (15) as well as the viscous correction $8.8 E^{1 / 2}$ determined in [13] for the first excited resonance in Io's configuration, but all our conclusions remain valid using prefactors of the same order of magnitude.

Formula (15) allows us to compute the order of magnitude of the growth rate of the elliptical instability in Io's core. We take as typical values an imposed magnetic field by Jupiter $B_{0}=1850 n T$, and for Io's core $R=$ $900 \mathrm{~km}, 2 \pi / \Omega_{0}=1.77$ days, and $\nu=10^{-6} \mathrm{~m}^{2} \mathrm{~s}^{-1}, \sigma_{e}=4 \times 10^{5} \mathrm{Sm}^{-1}, \rho=$ $12000 \mathrm{kgm}^{-3}$, consistent with a Fe/Fe-S composition. Then, $\mathrm{Rm}=1.7 \times 10^{7}$, $E=3.0 \times 10^{-14}, \Lambda=2.8 \times 10^{-6}$ and (15) implies that none of the elliptical modes is significantly affected by Joule damping. The typical growth rate of the tidal instability in Io is about 0.014 years $^{-1}$, suggesting rapid largescale variations in its core flows. Supposing that the spin-over mode still has an important component in Io's core (see figure 8 and appendix), its saturation amplitude would be about $\Omega_{H}=0.096 \Omega_{0}$ according to (8). The 
corresponding induced magnetic field would be a dipole aligned with the spinover axis and of typical amplitude $b_{r} \sim \sin (0.096) B_{0}=178 n T$, as derived from [16] in the relevant limit of large magnetic Reynolds number. We expect this field to fluctuate on rapid times ranging between the rotation period of 1.77 days and the typical time given by the growth rate of the instability of 72 years. The question then remains whether this field is measurable outside the core. Taking as in the Earth a typical mantle conductivity of $0.1-1 S . m^{-1}$ and considering the short period signal of about 1 day, we find a skin length of $200-600 \mathrm{~km}$. In the Earth, this low value compared to the typical depth of the mantle means that signals coming from the elliptical instability will be totally filtered. This will not be the case in Io. Hence, continuous field measurements of the ambient field in the vicinity of Io would allow to discriminate between its internal and atmospheric origins, an issue raised since the first punctual measurements provided by the Galileo mission (e.g. [24]).

\section{Conclusion}

In this paper, we have studied the effects of an imposed magnetic field on the elliptical instability in spheroids. By combining theoretical elements of previous works [16], [17], we have extended the nonlinear system governing the dynamics of the spin-over mode to include simultaneously the magnetic and viscous damping. We have shown theoretically and confirmed experimentally that the linear growth rate of the instability as well as the square amplitude of the induced magnetic dipole fall down linearly with the Elsasser number (i.e. with the square of the imposed magnetic field), with good agreement regarding predicted and measured prefactors. These conclusions have then been extended to all possible resonances of the elliptical instability using a short-wavelength Lagrangian approach. Applied to the specific case of Io in the magnetic field of Jupiter, we conclude that despite the viscous and Joule damping, a tidal instability is more than probable in the Jovian moon's core and induces in the core a relatively important field of about $10 \%$ of the ambient value. In addition to the magnetospheric interactions with Jupiter [25], we thus conclude from purely magnetohydrodynamical considerations that the elliptical instability provides a significant and non-stationary contribution to the magnetic field measured in the vicinity of Io, as first suggested by [13]. Continuous measurements in Io's vicinity should allow to discriminate 
between internal and external magnetic signatures.

This research was funded by a PHD-scholarschip of the Ministère de la Recherche Française, and by the ANR-IMAGINE (ANR-07BLAN-0182). We are also grateful to H. Bouchiat who kindly provided the Helmholtz coils.

\section{Appendix: WKB analysis of Io's tidal in- stability}

The now classical application of the short-wavelength "WKB" theory to inviscid fluids was developed in [9] and [21], generalized in [22] and [18], and summarized for the elliptical instability in [23]. It consists in looking for a perturbed solution of the full equations of motion under the form of a plane wave along the streamlines of the base flow. In our case, we thus look for a perturbed solution of the non-dimensional system

$$
\begin{array}{r}
\nabla \cdot \mathbf{u}=0, \\
d_{t} \mathbf{u}+(\mathbf{u} . \nabla) \mathbf{u}=-\nabla p+\frac{\Lambda}{R m}(\nabla \times \mathbf{b}) \times \mathbf{b}, \\
\nabla \cdot \mathbf{b}=0, \\
d_{t} \mathbf{b}+(\mathbf{u} . \nabla) \mathbf{b}=(\mathbf{b} . \nabla) \mathbf{u}+\frac{1}{R m} \nabla^{2} \mathbf{b},
\end{array}
$$

under the form

$$
\begin{aligned}
& \mathbf{u}=\mathbf{U}_{\mathbf{b}}+\mathbf{u}(t) e^{i \mathbf{k}(t) \cdot \mathbf{x}}, \\
& \mathbf{p}=\mathbf{P}_{\mathbf{b}}+\mathbf{p}(t) e^{i \mathbf{k}(t) \cdot \mathbf{x}}, \\
& \mathbf{b}=\mathbf{B}_{\mathbf{0}}+\mathbf{b}(t) e^{i \mathbf{k}(t) \cdot \mathbf{x}},
\end{aligned}
$$

along the streamlines of the base flow described by

$$
\frac{d \mathbf{x}}{d t}=\mathbf{U}_{\mathbf{b}},
$$

where $\mathbf{U}_{\mathbf{b}}$ stands for the two-dimensional base flow, $\mathbf{P}_{\mathbf{b}}$ for the corresponding pressure field, $\mathbf{B}_{\mathbf{0}}=(0,0,1)$ for the (non-dimensional) imposed vertical magnetic field and $\mathbf{x}$ for the position vector. The linearised MHD equations then write

$$
\mathbf{k} \cdot \mathbf{u}=0
$$




$$
\begin{aligned}
& d_{t} \mathbf{u}+i \mathbf{u}\left(d_{t} \mathbf{k} \cdot \mathbf{x}\right)+i\left(\mathbf{U}_{\mathbf{b}} \cdot \mathbf{k}\right) \mathbf{u}+(\mathbf{u} \cdot \nabla) \mathbf{U}_{\mathbf{b}}=-i \mathbf{k} p+\frac{\Lambda}{R m}(i \mathbf{k} \times \mathbf{b}) \times \mathbf{B}_{\mathbf{0}} \\
& \mathbf{k} \cdot \mathbf{b}=0 \text {, } \\
& d_{t} \mathbf{b}(t)+i \mathbf{b}\left(d_{t} \mathbf{k} \cdot \mathbf{x}\right)+i\left(\mathbf{U}_{\mathbf{b}} \cdot \mathbf{k}\right) \mathbf{b}=(\mathbf{b} \cdot \nabla) \mathbf{U}_{\mathbf{b}}+\left(\mathbf{B}_{\mathbf{0}} \cdot \mathbf{k}\right) \mathbf{u}-\frac{k^{2}}{R m} \mathbf{b},
\end{aligned}
$$

The velocity and induction equations can be decoupled in space and time to give an equation for the wave vector only

$$
d_{t} \mathbf{k} \cdot \mathbf{x}+\mathbf{U}_{\mathbf{b}} \cdot \mathbf{k}=0
$$

Linearized equations are then solved analytically using a perturbative expansion in the small parameter (i.e. the eccentricity in our case), supposing that the Elsasser number is of order 1. In this context, equations for fluid motions at order 0 are similar to the purely hydrodynamical equations, and the Lorentz force only induces a correction at order 1 . Technically, the easiest way to solve the MHD equations in our case is to use the vertical velocity $u_{z}$ and the vertical vorticity $W_{z}=\partial_{x} u_{y}-\partial_{y} u_{x}=i\left(k_{x} u_{y}-k_{y} u_{x}\right)$ of the perturbed field as unknowns, as well as the vertical component $b_{z}$ of the perturbed magnetic field and the corresponding magnetic vertical vorticity $C_{z}=i\left(k_{x} b_{y}-k_{y} b_{x}\right)$. The resolution is then straightforward.

The study of the $2 \mathrm{D}$ base flow $\mathbf{U}_{\mathbf{b}}$ given by (1), which corresponds to a stationary tidal deformation, closely follows the results already presented by Le Dizès [23]. From (28), one immediately finds the wave vector

$$
\mathbf{k}(t)=k\left(\frac{\sin (a)}{\sqrt{A}} \cos (\chi t), \sin (a) \sqrt{A} \sin (\chi t), \cos (a)\right)
$$

where $k$ is a constant, $A=\sqrt{(1+\epsilon) /(1-\epsilon)}$ is the ellipticity, $\chi=\sqrt{1-\epsilon^{2}}$, and $a$ is the angle between the flow rotation axis and the wavevector. Equations for fluid motions at order 0 give the expression of $\mathbf{u}$ at order 0 with a temporal frequency $f$

$$
f= \pm 2 \cos (a)
$$

whereas the linearised induction equation immediately gives the expression of $\mathbf{b}$ at order 0 . According to [23], an elliptical instability is possible if the forcing terms due to the elliptical deformation oscillate at the same frequency as the inertial wave, which means in our case $f=1$. Then, at order 1 in $\epsilon$, inertial waves resonate implying an exponential growth rate of the elliptical instability [23]

$$
\sigma_{n v}=\frac{9}{16} \epsilon-\frac{k^{4} \Lambda}{4\left(R m^{2}+k^{4}\right)} .
$$


This result is not directly applicable to the case of Io, where the elliptical deformation is not stationary. Indeed, Io is almost synchronized with Jupiter. It means that, in an isolated Jupiter-Io system, Io's spinning and orbital periods would be exactly equal: Io would always present the same face to Jupiter, and the tidal bulge would rotate at exactly the same frequency as Io. However, well-known orbital resonances with the other Galilean satellites maintain a 0.004 eccentricity in Io's orbit. The equality of orbital and spinning velocities is only true on average: in reality the orbital angular velocity hence the tidal bulge angular velocity - varies periodically with the orbital radius around this mean. Focusing on the first harmonic of this oscillation, the orbital angular velocity in the absolute frame of reference writes $1-\beta \cos (t)$, where $\beta \sim 0.008$ is twice the eccentricity of Io's orbit and where time is made dimensionless using Io's (constant) spin velocity. As demonstrated by Kerswell and Malkus [13], the fluid's laminar response in Io's core driven by this tidally distorted mantle motion corresponds in the bulge frame to the simple elliptical flow

$$
\mathbf{U}_{\mathbf{b}}^{B . F .}=(-(1+\epsilon) \beta \cos (t) Y,(1-\epsilon) \beta \cos (t) X, 0),
$$

which is an exact nonlinear solution to the incompressible Navier-Stokes equations of motion for any finite viscosity in the spheroid of equation $X^{2} /(1+$ $\epsilon)+Y^{2} /(1-\epsilon)+Z^{2}=1$. A simple change of frame then gives the base flow in the absolute frame of reference at first order in $\beta$

$$
\begin{aligned}
\mathbf{U}_{\mathbf{b}}= & (-y+\beta \epsilon \cos (t)(\sin (2 t) x-\cos (2 t) y), \\
& x-\beta \epsilon \cos (t)(\cos (2 t) x+\sin (2 t) y]), 0) .
\end{aligned}
$$

For a given initial position $(R, 0)$, streamlines are described by

$$
\begin{array}{r}
x(t)=R \cos (t)+\frac{\beta \epsilon R}{2}(1-\cos (2 t)), \\
y(t)=R \sin (t)-\frac{\beta \epsilon R}{2} \sin (2 t),
\end{array}
$$

(note that the results of the WKB theory do not depend on the chosen initial position along a closed trajectory). The solution to the wave vector equation along this streamline then writes

$$
\begin{aligned}
\mathbf{k}(t)=k \quad & (\sin (a) \cos (t+\phi)+\beta \epsilon / 2(\cos (2 t-\phi)-\cos (\phi)), \\
& \sin (a) \sin (t)+\beta \epsilon / 2(\sin (2 t-\phi)+\sin (\phi)), \cos (a)),
\end{aligned}
$$


where $k, a$ and $\phi$ are constant. At order zero in $\beta \epsilon$, the system can be reduced to a single equation for $u_{z}$

$$
\frac{d^{2} u_{z}^{0}}{d t^{2}}+4 \cos ^{2}(a) u_{z}^{0}=0
$$

whose solution writes

$$
u_{z}(t)=c_{1} e^{i f t}+c_{2} e^{-i f t}
$$

where $c_{1}, c_{2}$ are constant and $f$ is the frequency determined as a function of the wave vector, i.e. $f=2 \cos (a)$. At order 1 , we allow a long term variation of the solution at order zero, i.e.

$$
u_{z}(t)=\left(c_{1} e^{i f t}+c_{2} e^{-i f t}\right) e^{\beta \epsilon \sigma t},
$$

where $\sigma$ is the growth rate of the instability. The system then reduces to the same type of equation as (37), with a forcing term directly coming from the first order terms in the base flow. According to (33), terms of type $\cos (t) \sin (2 t)$ and $\cos (t) \cos (2 t)$ arise here, and the equation at order 1 schematically reads

$$
\frac{d^{2} u_{z}^{1}}{d t^{2}}+4 \cos ^{2}(a) u_{z}^{1}=F\left(e^{i t / 2}, e^{-i t / 2}, e^{3 i t / 2}, e^{-3 i t / 2}\right) .
$$

Solvability conditions then imply

$$
\begin{gathered}
\int_{0}^{2 \pi / f} F\left(e^{i t / 2}, e^{-i t / 2}, e^{3 i t / 2}, e^{-3 i t / 2}\right) e^{i f t}=0 \\
\int_{0}^{-2 \pi / f} F\left(e^{i t / 2}, e^{-i t / 2}, e^{3 i t / 2}, e^{-3 i t / 2}\right) e^{-i f t}=0
\end{gathered}
$$

which validate the whole asymptotic approach by ensuring that forcing terms are not secular. This system with unknown $c_{1}, c_{2}$ admits a non trivial solution if and only if $f=1 / 2$ or $f=3 / 2$, in which case the growth rate $\sigma$ is determined by the nullity of the determinant. It is then maximized over all values of wavevector phase $\phi$. The maximum is obtained for $f=1 / 2$ and $\phi=\pi / 4$ and writes

$$
\sigma=\frac{17}{64} \sqrt{(\beta \epsilon)^{2}-\frac{576}{289} \frac{\Lambda^{2} R m^{2} k^{4}}{\left(R m^{2}+4 k^{4}\right)^{2}}}-\frac{3}{4} \frac{k^{4} \Lambda}{R m^{2}+4 k^{4}} .
$$


Note that the resonance condition $f=1 / 2$ corresponds to the resonance condition for the closely related precession instability, where the spin-over mode is also known to be excited [11]. This validates the evaluation of the spin-over induction performed at the end of section 5 .

\section{References}

[1] Leweke, T. \& Williamson, C. H. K. , 1998, Cooperative elliptic instability of a vortex pair. J. Fluid Mech. , 360, 85-116.

[2] Meunier, P. \& Ehrenstein, U. \& Leweke, T. \& Rossi, M. , 2002, A merging criterion for two-dimensional co-rotating vortices. Phys. Fluids, 14, 2757-2766.

[3] Malkus, W. V. R., 1989, An experimental study of global instabilities due to tidal (elliptical) distorsion of a rotating elastic cylinder. Geophys. Astrophys. Fluid Dyn., 48, 123-134.

[4] Eloy, C. \& Le Gal, P. \& Le Dizès, S., 2003, Elliptic and triangular instabilities in rotating cylinders. J. Fluid Mech., 476, 357-388.

[5] Lacaze, L. \& Le Gal, P. \& Le Dizès, S., 2004, Elliptical instability in a rotating spheroid. J. fluid Mech. , 505, 1-22.

[6] Lacaze, L. \& Le Gal, P. \& Le Dizès, S., 2005, Elliptical instability of a flow in a rotating shell. Phys. Earth Planet. Int. , 151, 194-205.

[7] Pierrehumbert, R. T. , 1986, Universal short-wave instability of twodimensional eddies in an inviscid fluid. Phys. Rev. Lett. , 57, 21572160 .

[8] Kerswell, R.R., 2002, Elliptical instability, Annual Review of Fluid Mechanics, 34, 83-113.

[9] Bayly, B. J., 1986, Three-dimensional instability of elliptical flow. Phys. Rev. Lett. , 57, 2160-2163.

[10] Waleffe, F., 1990, On the three-dimensional instability of strained vortices. Phys. Fluids A , 57, 76-80.

[11] Malkus, W. V. R., 1968, Precession of the earth as the cause of geomagnetism. Science, 160, 259-264

[12] Kerswell, R. R., 1994, Tidal excitation of hydromagnetic waves and their damping in the Earth. J. Fluid Mech., 274, 219-241. 
[13] Kerswell, R.R. \& Malkus, W.V.R., 1998, Tidal instability as the source for Io's magnetic signature. Geophys Res Lett, 25, 603-606.

[14] Aldridge, K. D. \& Lumb, L. I., 1987, Inertial waves identified in Earth's fluid outer core. Nature, 325, 421-423.

[15] Tilgner, A., 2005, Precession driven dynamos. Phys. Fluids, 17, 034104 .

[16] Lacaze, L. \& Herreman W. \& Le Bars M. \& Le Dizès, S. \& Le Gal, P., 2006, Magnetic field induced by elliptical instability in a rotating spheroid. Geophys. Astrophys. Fluid Dyn. , 100, 299-317.

[17] Thess, A. \& Zikanov, O. , 2007, Transition from two-dimensional to three-dimensional magnetohydrodynamic turbulence. J. fluid Mech. , 579, 383-412.

[18] Lifschitz, A. \& Hameiri, E., 1991, Local stability conditions in fluid dynamics. Phys. Fluids, 3, 2644-2651.

[19] Le Bars, M. \& Le Dizès, S. \& Le Gal, P. , 2007, Coriolis effects on the elliptical instability in cylindrical and spherical rotating containers. $J$. Fluid Mech., 585, 323-342.

[20] Greenspan, H. P., 1968, The Theory of Rotating Fluids. Cambridge University Press, London.

[21] Craik, A. D. D. \& Criminale, W. O., 1986, Evolution of wavelike disturbances in shear flows - a class of exact solutions of the Navier-Stokes equations. Proc. Roy. Soc. A406, 13-26.

[22] Friedlander, S. \& Vishik, M., 1991, Instability criteria for steady flows of a perfect fluid. Phys. Rev. Lett., 66, 2204-2206.

[23] Le Dizès, S., 2000, Three-dimensional instability of a multipolar vortex in a rotating flow. Phys. Fluids, 12, 2762-74.

[24] Kivelson, M. G. \& Khurana, K. K. \& Russell, C. T. \& Joy, Steven P. \& Volwerk, M. \& Walker, R. J. \& Zimmer, C. \& Linker, J. A. , 2001, Magnetized or unmagnetized: Ambiguity persists following Galileo's encounters with Io in 1999 and 2000 J. Geophys. Res., 106, 2612126136.

[25] Saur, J. \& Neubauer, F. \& Connerney, J. E. P. \& Zarka, P. \& Kivelson, M. G., 2004, Plasma interaction of Io with its plasma torus. In 
Jupiter. The planet, satellites and magnetosphere. Edited by F. Bagenal, T. E. Dowling, W. B. McKinnon. Cambridge planetary science, Vol. 1, Cambridge, UK: Cambridge University Press, ISBN 0-52181808-7, 537-560. 

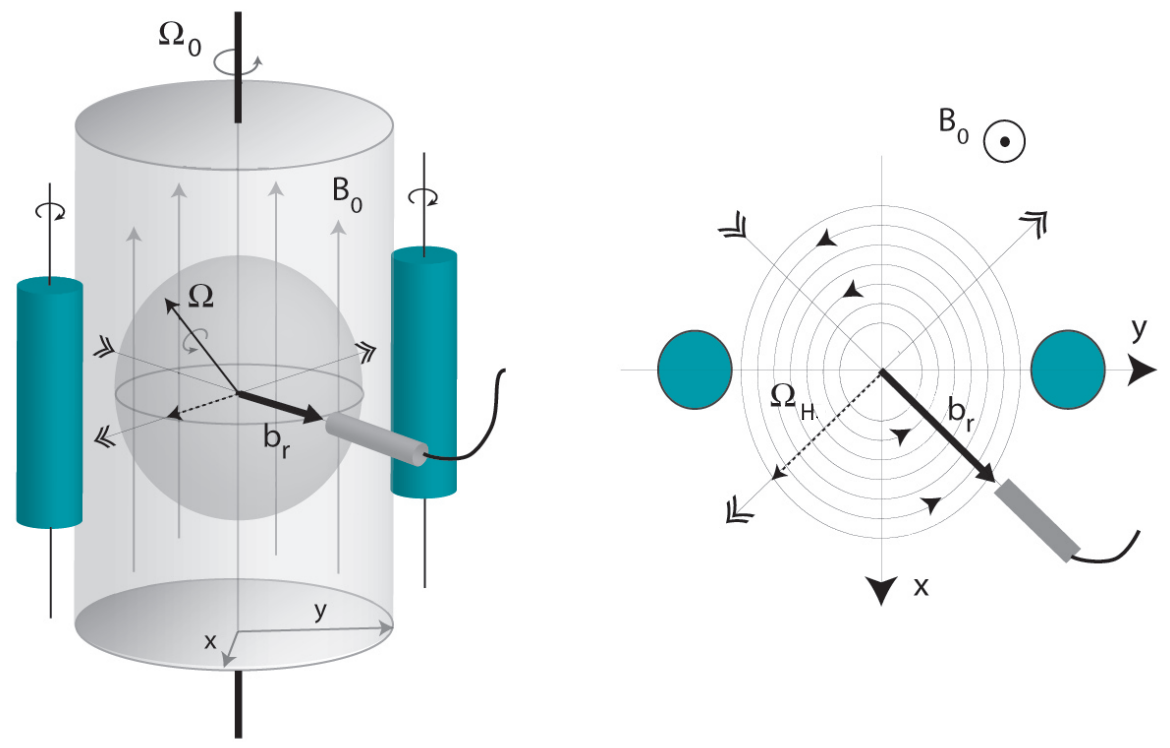

Figure 1: Sketch of the set-up, side and top-view (see also Lacaze et al. [16]). A liquid metal in a deformable spheroidal cavity rotates at $\Omega_{0}$. A strong magnetic field $B_{0}$ is imposed along the rotation axis. Fixed rollers induce an elliptical deformation of the streamlines. Also shown here is the horizontal projection of the spin-over mode, corresponding to a transverse solid body rotation $\Omega_{H}$ in the stretched direction (dashed arrow), which tilts the rotation axis of the fluid to $\Omega$. In the limit of low magnetic Reynolds number, $\Omega_{H}$ induces in the compressed direction a dipolar magnetic field $b_{r} \sim \Omega_{H}$, which is measured by a Hall probe. 

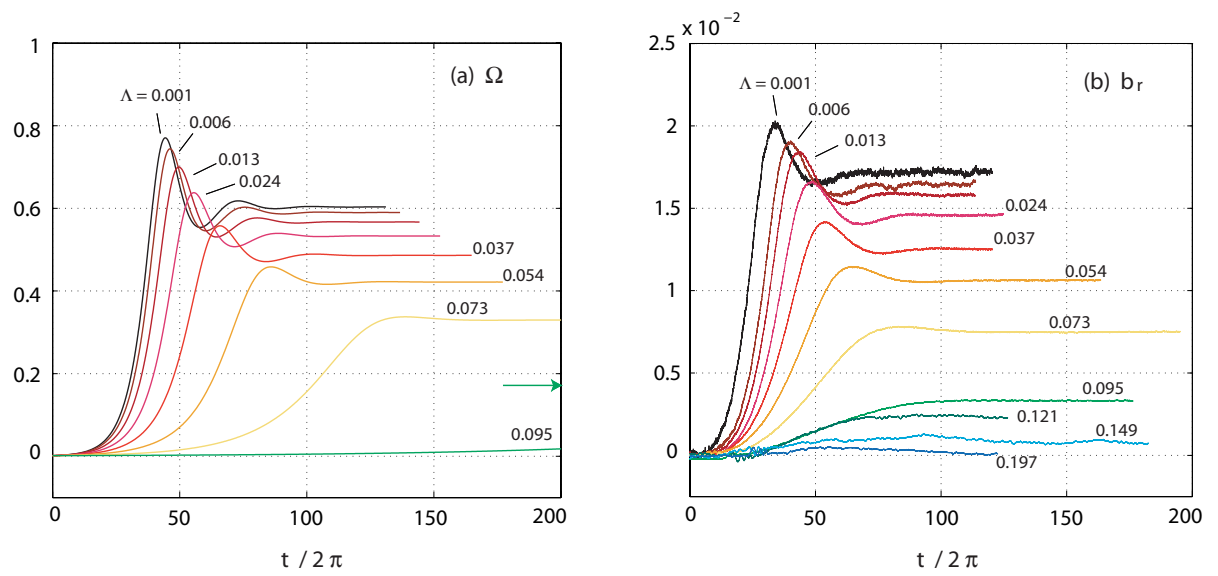

Figure 2: (a) Theoretical nonlinear temporal evolution of the horizontal projection of the spin-over mode amplitude $\Omega_{H}=\left(\Omega_{1}^{2}+\Omega_{2}\right)^{1 / 2}$, for various values of the Elsasser number $\Lambda$. Ekmann number and eccentricity are fixed, $E=8.53 \times 10^{-5}, \epsilon=0.10$. Calculations started from the initial state $\Omega_{1}=10^{-3}, \Omega_{2}=-10^{-3}, \Omega_{3}=0$, which is the linearly unstable spin-over mode with small amplitude. The arrow on the right side, indicate the saturation level of the slowly growing spin-over mode horizontal amplitude at $\Lambda=0.095$. The critical Elsasser number is $\Lambda^{c}=0.103$ for this parameter set. (b) Typical recorded magnetic field-signals for varying Elsasser number. Ekmann number and eccentricity are fixed $E=8.53 \times 10^{-5}, \epsilon=0.10 \pm 0.005$ . The experiments agree with the theoretical profiles of figure $2(\mathrm{a})$. 


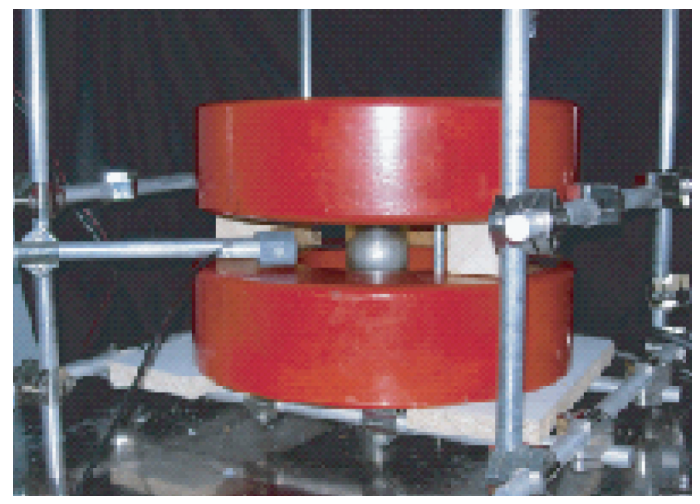

Figure 3: Picture of the experimental set-up. Large watercooled Helmholtz coils provide a homogenous magnetic field up to $B_{0}=0.1 \mathrm{~T}$. Induced fields are measured with a radial Hall probe.

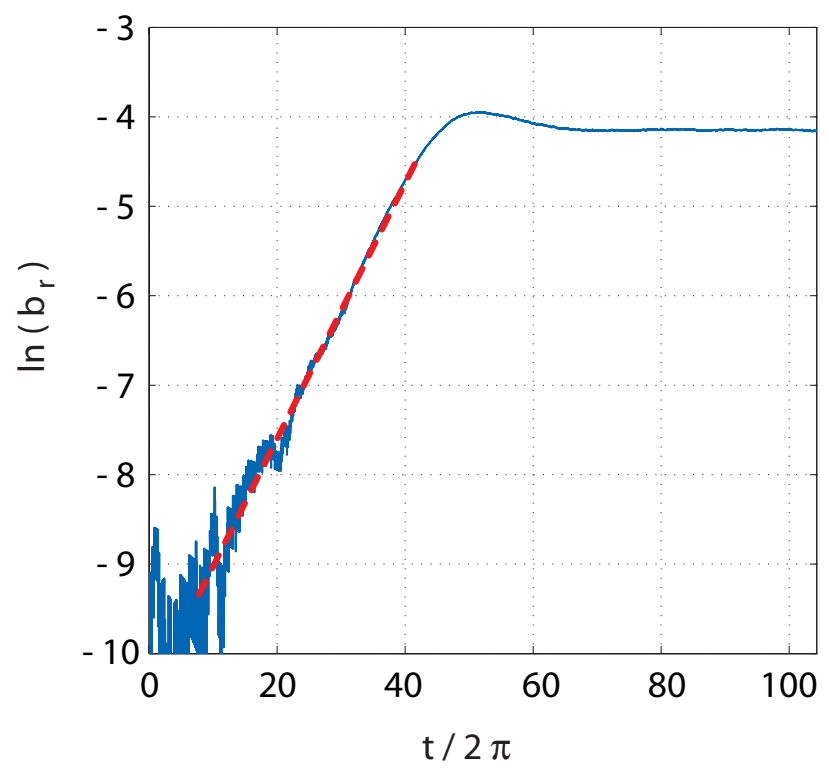

Figure 4: Logarithm of the induced magnetic field signal (full line) at $E=$ $8.53 \times 10^{-5}, \epsilon=0.100 \pm 0.005, \Lambda=0.01$. The slope of the linear fit (dashed line) provides the initial linear growth rate. 


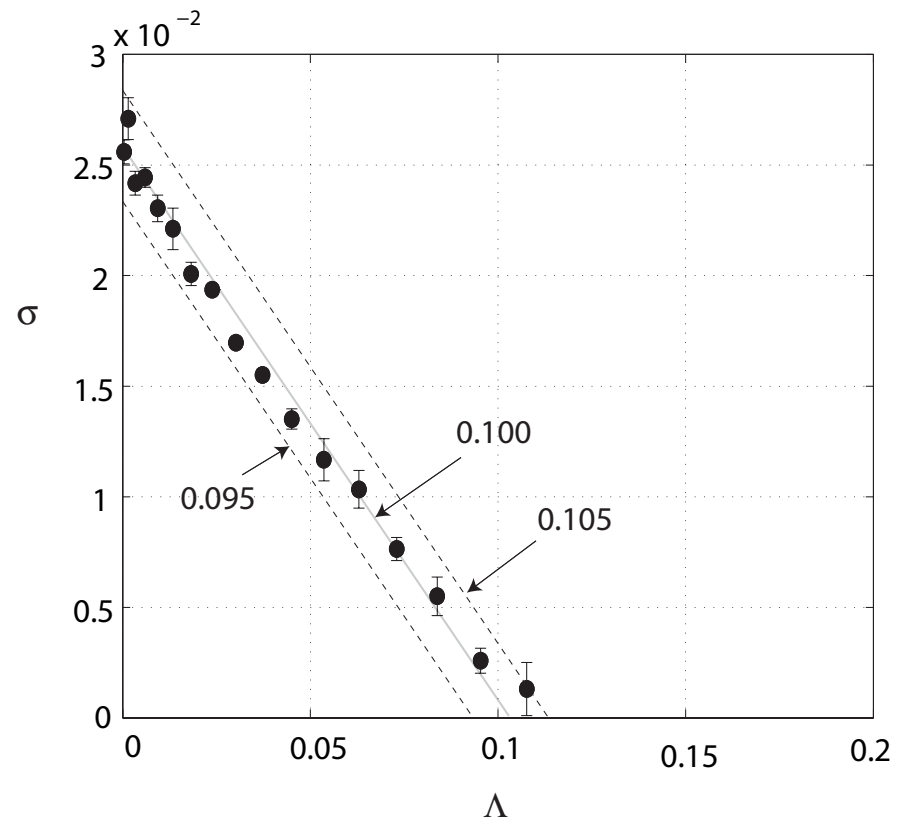

Figure 5: Linear growth rates $\sigma$ as a function of Elsasser number $\Lambda . E=$ $8.5 \times 10^{-5}, \epsilon=0.100 \pm 0.005$. The experimental measurements (•) are in good agreement with the theoretical values for $\epsilon=0.10$ (soft line). Also shown are the theoretical (dashed) curves for $\epsilon=0.095$ and $\epsilon=0.105$, representing the uncertainty in $\epsilon$; note however that there is no adjusting parameter in the comparison between theory and experiment. Growth was no longer exponential beyond $\Lambda \geq 0.121$. 


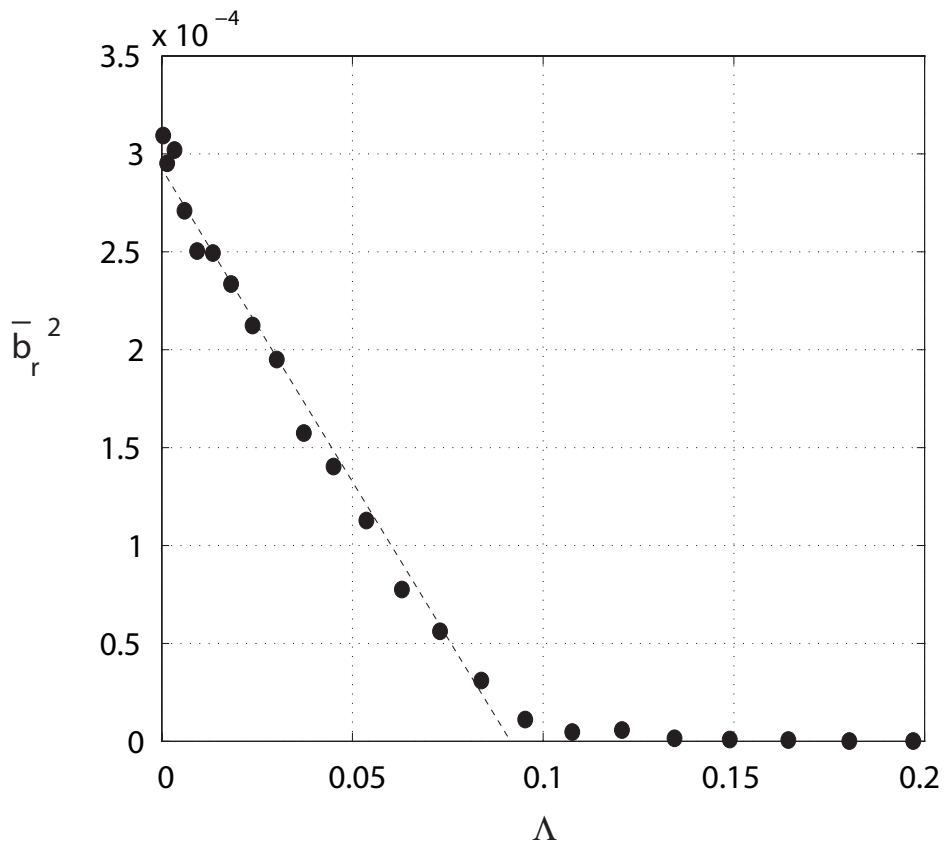

Figure 6: Square of the measured saturation-amplitudes of the magnetic field $b_{r, s a t}^{2}$, as a function of Elsasser number $\Lambda . E=8.5 \times 10^{-5}, \epsilon=0.10$. Experimental measurements $(\bullet)$ and linear fit (dashed line). The saturation amplitudes are in agreement with the weakly nonlinear scaling, predicting a linear dependance on $\Lambda$. 


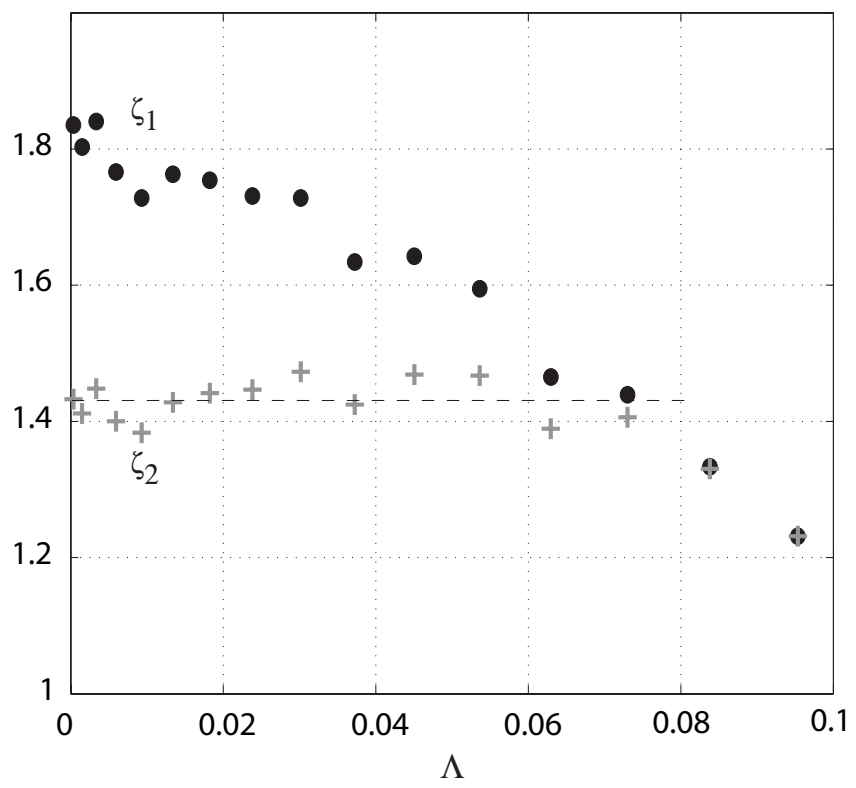

Figure 7: Ratios of the experimentally observed saturation amplitude, to the theoretical saturation amplitudes $\zeta_{1}(\bullet)$ and to the maximum amplitude attained during the overshoot $\zeta_{2}(+)$, as a function of the Elsasser number

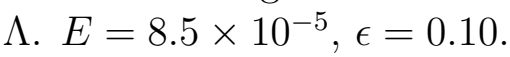



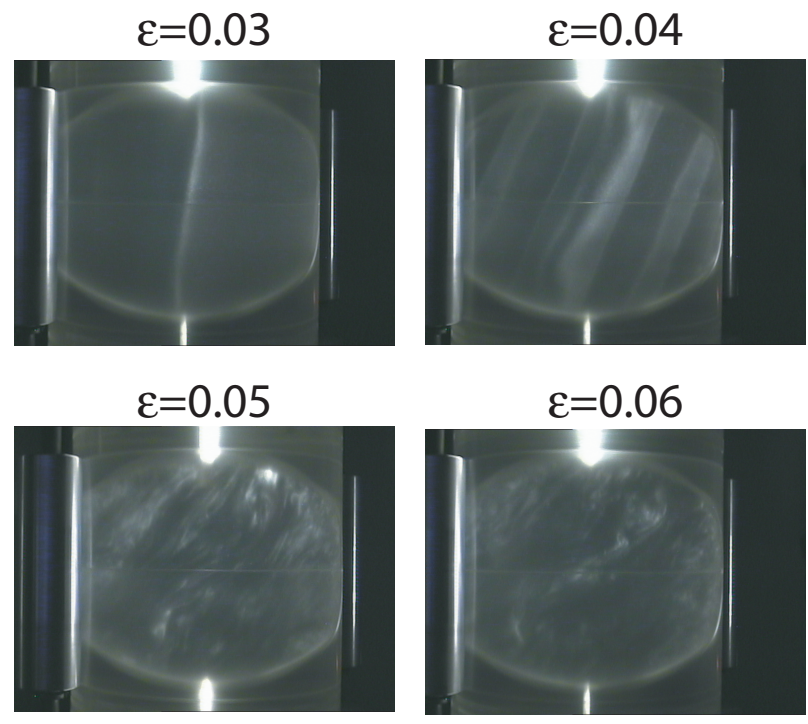

Figure 8: Kalliroscope visualization of the elliptical instability for a fixed Ekmann number $E=10^{-5}$ and increasing values of $\epsilon$ (purely hydrodynamical experiment). As suggested by (6), the relevant parameter to describe the dynamics of the elliptical instability is $\alpha=E^{1 / 2} / \epsilon$. Decreasing $\alpha$ from 0.11 to 0.053 , the flow becomes more and more complex, especially at small scale, but the spin-over mode remains present at large scale. The same behavior is expected to remain valid at the planetary scale, for instance in Io's core where $\alpha \sim 0.0036$. 